\title{
What is the time course of working memory attentional refreshing?
}

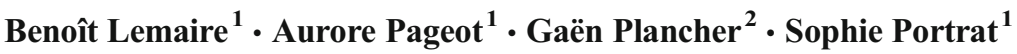

Published online: 31 March 2017

(C) Psychonomic Society, Inc. 2017

\begin{abstract}
One way of maintaining information in working memory is through attentional refreshing, a process that was recently shown to be independent from verbal rehearsal. In the classical working memory complex span task, the usual assumption is that memoranda are refreshed in a cumulative fashion, starting from the first item, going in a forward order until the latest one, and cycling until there is no time to continue the process. However, there is no evidence that refreshing operates in that way. The present study proposes a computational modelling study, which constitutes a powerful method to investigate alternative hypotheses. Different refreshing schedules are investigated within computational implementation of the time-based resource sharing model. Their ability to fit three sets of behavioral data and to reproduce the major time-based resource sharing predictions were evaluated using standard model selection criteria. Besides an already published schedule in which the attentional focus is expanded, it appeared that one schedule, the least-activated-first, outperforms the classical cumulative one. The memory trace refreshed at a given time is the one that is the least activated in working memory at that time. These findings characterized the time course of attentional refreshing in working memory and specified the contribution of refreshing to primacy and recency effects. Moreover, in the light of various fields of
\end{abstract}

Benoît Lemaire

Benoit.Lemaire@univ-grenoble-alpes.fr

1 Univ. Grenoble Alpes, CNRS, LPNC UMR 5105, F-38000 Grenoble, France

2 Univ. Lyon 2, EMC, 5 avenue Pierre Mendès-France, F-69676 Bron, France cognitive psychology, we propose that such refreshing schedules can operate without a homunculus within a general framework including cognitive control and strategic considerations.

Keywords Working memory · Computational modeling · Attentional refreshing

Working memory (WM) is a system devoted to the immediate storage of information that we all use in our daily life to maintain information for short periods of time while performing other activities. Because of forgetting, a specific maintenance mechanism called refreshing has been proposed to preserve memory traces and hence to allow the recall of previously encoded items (Barrouillet \& Camos, 2015; Cowan, Saults, \& Elliott, 2002; Hudjetz \& Oberauer, 2007; Johnson, 1992; Johnson et al., 2005; Raye, Johnson, Mitchell, Greene, \& Johnson, 2007). For several years, different studies based on behavioral or neuroimaging data have tried to describe this phenomenon (e.g., Raye et al. 2007; Vergauwe, Camos, \& Barrouillet, 2014; Vergauwe \& Cowan, 2015). A solid understanding of refreshing is crucial because decay-based theoretical models of WM are grounded in this mechanism. The present study therefore proposes a complementary approach, computational modeling, with the aim of specifying the mechanism of refreshing through the simulation of its time course within a classical WM task.

Such a task is typically a serial recall task, where participants are presented with items that they have to recall, in forward order, at the end of the trial. To study more precisely how items are maintained in memory throughout the trial, distracting tasks are intertwined with the presentation of items, to form the so-called complex span task (CST). A simple 
example of such a task consists of presenting a sequence of letters and digits to be read (e.g., one every second, participants being asked to recall only letters, in forward order, at the end of the trial). The task could be extended in many ways, for example, by varying the number of items to be remembered, the type of items (letters, symbols, images, etc.), the type of distractors and the nature of the distracting task (visual search, reading aloud of letters or words, naming images, etc.), the number of distractors, the degree of similarity between items and distractors, the repetition or not of distractors, and the duration of presentation of items.

Several kinds of theoretical models account for human performance in such CST. Among them, decay-based models (e.g., Anderson, Reder, \& Lebière, 1996; Barrouillet \& Camos, 2015) presume that items are represented along with a level of activation that directly influences the probability of recall. Attention plays a major role in these models; when attention is not directed to an item, for example, because of processing a distractor or encoding another item, its level of activation decays. When attention is not occupied by performing a distracting task or by encoding items, the free time is proposed to be used to refresh items, namely, pulling up activation values of items to be maintained. It is worth noting that other WM theoretical models deny the existence of decay-based forgetting in WM and rather consider that memory loss is due to interference (e.g., Oberauer, Lewandowsky, Farrell, Jarrold, \& Greaves, 2012). However, this study does not enter this debate, and the current trend is to consider that both decay and interference actually occur (Altmann \& Schunn, 2012). The work presented here depends on this decay-based view and focuses on the specification of the refreshing mechanism.

Actually, there are two mechanisms for compensating the decay of verbal memory traces. The first one is phonological rehearsal (Baddeley, 1986). This consists of the conscious articulatory repetition of the verbal items to be maintained, vocally or subvocally, in a forward-order loop as long as there is time to do this. As mentioned above, it has been proposed that a second mechanism, usually called refreshing, is used by participants (Camos \& Barrouillet, 2014; Cowan et al., 2002; Hudjetz \& Oberauer, 2007; Raye et al., 2007). This is described as a general attention-based maintenance mechanism (Barrouillet, Portrat, \& Camos, 2011; Camos, Mora, \& Oberauer, 2011; Cowan, 1999, 2005; Johnson, 1992; Johnson et al., 2005; Raye et al., 2007). A precise definition of refreshing is still not consensual nowadays. Nonetheless, we will see that it could be considered as an executive function, "one of the most elementary, but critical, components of reflection" (Johnson et al., 2002, p. 64), which operates in $\mathrm{WM}$ as an additional maintenance mechanism (Camos \& Barrouillet, 2014) by retrieving previously presented information through attentional focusing (Loaiza \& McCabe, 2012; Vergauwe \& Cowan, 2015).
The idea of two forms of verbal maintenance mechanisms was already proposed during the development of Baddeley's multicomponent model (Vallar \& Baddeley, 1982), but this is only recently that evidence for another mechanism, attentional and independent from rehearsal, has been provided (see a review by Camos \& Barrouillet, 2014). For instance, in Camos, Lagner, and Barrouillet (2009), the opportunity of using one of the maintenance mechanisms was manipulated while the other was impeded in complex span tasks. In an experiment, the phonological rehearsal was impeded by a concurrent articulation whereas the availability of attentional refreshing was varied by a distracting task that was more or less attention demanding (e.g., reading vs. solving operations). As expected, although participants recalled a substantial amount of memory items, reducing the availability of one mechanism while the other was impeded had a detrimental effect on recall performance. Moreover, it was possible to observe the classical phonological similarity, or word length effects in the one hand or a cognitive load effect on the other hand, depending on whether participants were instructed to maintain items through either phonological rehearsal or attentional refreshing respectively (Camos et al., 2011). These findings, and others in the same vein (Camos, Mora, \& Barrouillet, 2013; Mora \& Camos, 2013) support the idea that verbal information can be maintained in WM by both attentional and phonological mechanisms both dealing with different features of the tobe-maintained information.

Another set of evidence for the dissociation between those two processes comes from neuroimaging studies. While articulatory rehearsal has been shown to recruit speech-related cortical regions, such as Broca's area and premotor cortex (Smith \& Jonides, 1997), refreshing corresponds to the intervention of the dorsolateral prefrontal cortex (Raye et al., 2007; Raye, Johnson, Mitchell, Reeder, \& Greene, 2002).

The specific role of refreshing, compared to the phonological mechanism, has been clarified by Marcia Johnson's work. In her MEM (multiple-entry, modular) memory model, she described a WM framework in which several processes allowed people to sustain, organize, and retrieve information. Among these, refreshing permits the reactivation of memory representation, whereas rehearsal refers to subvocal articulation (Johnson, 1992). Through a simple and clever paradigm, a condition requiring refreshing is contrasted with conditions involving other processes, mostly reading or repeating (Johnson et al., 2002; Johnson, Mitchell, Raye, \& Green, 2004; Johnson et al., 2005; Raye et al., 2002). For example, participants were first presented with single words that they had to read silently. Some of the words were followed by the same word (repeat condition) and others by a dot, signaling participants to think of the immediately previous word (refresh condition). The results consistently showed a longterm memory benefit from refreshing information in contrast to repeated information (e.g., Johnson et al., 2002). These 
results could be linked to the death of processing theory (Craik \& Lockhart, 1972): The deeper the encoding, the better the recall. Actually, a deep encoding involving controlled attention such as refreshing should lead to more stable memory traces than a superficial mechanism dealing only with phonological features.

In the specific context of WM complex span tasks, it has been shown that the more refreshing opportunities an item receives, the more likely it is to be recalled from episodic memory during a delayed recall (Camos \& Portrat, 2015; Loaiza \& McCabe, 2012; McCabe, 2008). To verify that this beneficial long-term effect of refreshing was not related to any maintenance mechanism, Camos and Portrat (2015) also manipulated the availability of phonological rehearsal. While the increase of refreshing opportunities leads to better recall in both immediate and delayed tests, the addition of concurrent articulation reduced immediate but not delayed recall. This study again suggests that an additional maintenance mechanism leading to stable and durable memory coexists with the well-known articulatory rehearsal, which concerns ephemeral phonological features.

Very recently, it was shown that refreshing is akin to memory search, both relying on a "basic covert memory process that quickly retrieves the memory items into the focus of attention, thereby reactivating the information" (Vergauwe \& Cowan, 2015, p. 1001). The authors used a WM complex span task, in which participants had to memorize a series of red letters while concurrently performing a processing task on intertwined black letters. This task consisted of judging the location of the letter (upper or lower part of the screen), judging the alphabetic position of the letter (before or after the letter $O$ ) or, in a memory condition, judging if each black letter was present in, or absent from, the red list presented so far. Because the alphabetic task prolonged attentional capture compared to the location task, poorer recall performance was expected and indeed was observed in this particular condition. However, even if the memory condition would also prolong attentional capture compared to the location condition, such forgetting was not expected and indeed was not observed. These findings suggested that memory items are reactivated during the memory search required for the memory condition of the processing task, as if those periods of time were directly used for refreshing per se.

Several findings thus support the existence of an attentional maintenance mechanism called refreshing, but the question of how items are refreshed is open. Regarding phonological rehearsal, except under times of high pressure, it appears that participants tend to rehearse verbal items using a cumulative forward-order strategy, starting from the first item on the list and proceeding in order until the last item encoded so far is reached (Tan \& Ward, 2008). However, the strategy for refreshing is much less clear because it is a very short covert process that is harder to study. Several attempts have been made to estimate the time needed to refresh a single item. In a WM paradigm, when participants are allowed to postpone the processing of a distractor, Vergauwe et al. (2014) found that each additional item in the memoranda extended this postponement by about 40 to $50 \mathrm{~ms}$. In the same way, the study of Jarrold, Tam, Baddeley, \& Harvey (2011) estimated the refreshing duration of a given item to be about $41 \mathrm{~ms}$, because they observed a linear trend between the distractor response time and the position of this processing episode in the memory list. Finally, Vergauwe and Cowan (2015) also found that refreshing would take about $40 \mathrm{~ms}$.

However, these estimates of refreshing duration are not informative about the way this refreshing mechanism takes place. The aforementioned behavioral studies depend on a basic assumption: the refreshing mechanism acts just as the rehearsal mechanism described in Baddeley's model (Baddeley \& Hitch, 1974). When participants have enough time, they engage in sequential and cyclical attentional refreshing, focusing on the items to be maintained, starting from the first item on the list and going in a forward order until the last one and cycling until there is no more time (Loaiza \& McCabe, 2012; McCabe, 2008). This hypothesis is sometimes taken for granted, as, for example, in Vergauwe et al. (2014): "Because attentional maintenance proceeds in a cumulative fashion, maintenance-based postponement of processing should linearly increase with the number of items to be maintained" (p. 1073). Unfortunately, as far as we know, there is no direct evidence that refreshing acts as such a cumulative mechanism. The goal of the present study is thus to consider alternative schedules, using a computational modeling approach to provide evidence for the time course of refreshing. To this end, we simulated the effects of different refreshing strategies and compared the outcomes to published behavioral data collected through experimental studies in adults (Barrouillet, Portrat, \& Camos, 2011; Barrouillet, Portrat, Vergauwe, Diependaele, \& Camos, 2011) as well as to the predictions of a well-known time-based WM theory, timebased resource sharing (TBRS; Barrouillet \& Camos, 2015).

The TBRS model is particularly suitable for such investigation because it is "the first attempt to specify the processes underlying complex span performance" (Oberauer \& Lewandowsky, 2011, p. 11), the complex span task being the best framework to study attentional refreshing as indicated above. In addition, TBRS has been described in a computational model (TBRS*; Oberauer \& Lewandowsky, 2011), which is the only attempt to implement closely the details of the verbal theory. Although TBRS* is rather recent, its parameters have been tested several times on different experimental data and showed reliable stability. Initially, Oberauer and Lewandowsky (2011) tested their model on seven different datasets. Portrat and Lemaire (2015) relied on another set of experimental data. Portrat, Guida, Phénix, and Lemaire (2016) applied TBRS* to data involving chunking, and Hoareau, 
Lemaire, Portrat, and Plancher (2016) used TBRS* to account for WM aging. All of these studies converged on the same set of parameters except for the study of Hoareau et al. (2016), where some parameters had to be modified with respect to the particular aging population examined. The current TBRS* parameters can therefore be considered as steady.

TBRS* implements a cyclical cumulative forward-order refreshing mechanism, which resembles rehearsal, and sets the duration for refreshing a single item to an average of 80 $\mathrm{ms}$. The authors mentioned that they considered other refreshing strategies but found that these were not satisfactory, meaning that none of them showed a better simulation of the human performance than cumulative refreshing. They tested a procedure which, for each new refreshing episode, continued with the next list item instead of the first list item and started over with the first item only after encoding a new item. This did not improve the model. These authors also tested a refreshing strategy that "focuses on the last encoded item" (Oberauer \& Lewandowsky, 2011, p. 21) as well as a random strategy that picks items with equal probability. None of these possible implementations of refreshing was satisfying, because they do not reproduce the primacy effect. The authors eventually considered a procedure that refreshes only those items that were below maximal activation but found no difference compared to the current cumulative forward-order strategy, which is the hypothesis that was finally proposed.

However, recent findings suggest a need to reconsider that cumulative strategy (Portrat \& Lemaire, 2015). Computational simulations with the TBRS* implementation containing a cumulative forward-order strategy predict that, under time pressure, the last items cannot be considered in the refreshing cycle. Even under major time pressure, when there are just a few hundreds of milliseconds between distracting episodes in a CST, adult participants are still able to recall the last items of a list, corresponding to the wellknown recency effect. For example, Barrouillet, Portrat, Vergauwe, et al. (2011) present the results of an experimental condition in which participants had to maintain up to seven items while performing a visually distracting task every 790 $\mathrm{ms}$, which needed an average of $400 \mathrm{~ms}$ to be performed, leaving less than $400 \mathrm{~ms}$ to refresh items. TBRS* predicts that the last items would be poorly recalled, because they would hardly be refreshed, compared to the first items which would be constantly refreshed at the beginning of each refreshing opportunity. Behavioral data run counter to this prediction (Portrat \& Lemaire, 2015): Participants showed around 50\% of correctly recalled items at Positions 5 and 6, whereas the predictions of the model implementing the cumulative forward-order refreshing were much lower and forecast almost $0 \%$ at Position 6.

One reason the model fails to reproduce the recency effect in such rapid conditions is the duration needed to refresh a single item, which is set to an average of $80 \mathrm{~ms}$ (Oberauer \&
Lewandowsy, 2011) and thus generally prevents the sixth item from being refreshed if the free time is about $5 \times 80=400 \mathrm{~ms}$. As presented above, recent experimental results suggest a refreshing time of about $40 \mathrm{~ms}$, but this shorter value is not long enough to reproduce the recency effect. The model needs to be adjusted, with a duration for refreshing a single item of $10 \mathrm{~ms}$ to account for the data (Portrat \& Lemaire, 2015). This is neither cognitively plausible, nor supported by behavioral data. Therefore, it is likely that participants do not engage in a cyclical sequential forward-order strategy at each refreshing opportunity.

A first alternative is to consider that several items are refreshed at the same time, within a larger attentional focus, an idea that has been implemented (Portrat \& Lemaire, 2015) and which is in line with Cowan's (2005) proposal of a flexible focus of attention. A cumulative forward-order refreshing mechanism performed within a focus of attention that can handle four items at the same time has demonstrated its ability to reproduce behavioral data (Portrat \& Lemaire, 2015). However, this new model was compared to data obtained in a CST where participants performed the processing task silently. Hence, as mentioned above, recall performance could have been mediated by both rehearsal and refreshing. Therefore, a refreshing mechanism based on focusing on more than one unit at a time cannot be taken for granted. It has to be tested with other behavioral data and compared with other possible schedules. To our knowledge, only one attempt has investigated different schedules to date (Oberauer \& Lewandowsky, 2011). Nevertheless, these authors did not simulate all the schedules they presented, ruling out some of them on purely theoretical grounds. Also, they suggested that "the onus is now on decay theorists to propose a schedule that brings their theory in line with the data" (Oberauer et al., 2012, p. 797). By implementing different refreshing mechanisms within the TBRS* model, the present study proposes to fill this gap.

Several other refreshing mechanisms are thus explored. Our approach was to implement these refreshing mechanisms within the TBRS* default model and to evaluate their ability to (a) fit behavioral data already published (Barrouillet, Portrat, \& Camos, 2011; Barrouillet, Portrat, Vergauwe, et al., 2011) and (b) reproduce the major TBRS predictions through a fictional set of experiments that were proposed by Oberauer and Lewandowsky (2011, Simulation 1).

\section{Alternative options for the time course of the refreshing mechanism}

To accurately predict behavioral data, and especially the recency effect in rapid conditions, the refreshing mechanism should restore the balance between items of a given series, which is the main defect of the cumulative approach because this gives too much weight to the first items. Here are the 
seven refreshing schedules that we considered (the first four being derived from Oberauer \& Lewandowsky's, 2011, proposal).

\section{Cumulative forward-order refreshing}

This is the mechanism presented above and implemented by Oberauer and Lewandowsky (2011). When there is free time, positions are considered in turn, starting with the first one. For each position, an item is retrieved and refreshed during a fixed period of time. If there is still free time after all the positions have been considered, another cycle starts.

\section{Random refreshing}

Since it appeared that the drawback of the sequential model is a fault in refreshing the last items, we defined a baseline strategy that would cause refreshing to operate uniformly over the different items. At each refreshing opportunity, a position is randomly selected, the corresponding item is retrieved and refreshed for a fixed time, then another position is randomly picked, and so on, until there is no time left because a new distracting task has to be performed or a new item has to be encoded. Oberauer and Lewandowsky (2011) implemented such a random strategy, picking items with equal probability and found it unsatisfactory because it did not reproduce the primacy effect.

\section{Last encoded item refreshing}

Oberauer and Lewandowsky (2011) suggested a schedule in which only the last item encoded is refreshed. From a theoretical point of view, it is obvious that the primacy effect should disappear with such a schedule, but we implemented it even so.

\section{Below activation threshold refreshing}

Oberauer and Lewandowsky (2011) also considered a schedule that would refresh only those items that are below maximal activation. Since activation follows an asymptote that by definition cannot be reached, there is no maximal activation value. We therefore considered different thresholds under which refreshing is performed. When all activation values are over the threshold, the model enters into the cumulative mode for one cycle. Oberauer and Lewandowsky ruled out this schedule, arguing that it would not be different from the classical cumulative strategy, because items whose strength is close to the asymptote do not gain further strength from refreshing, whether they are skipped or not. This is true; but not refreshing an item would save time for refreshing other items, which could make a difference, especially when there is not much time for refreshing. We implemented this possibility.

\section{Least activated first (LAF) refreshing}

This refreshing mechanism would first consider the position whose corresponding item is the most likely to be forgotten (i.e., the one whose activation value is minimal). The idea is that the least activated item has priority for refreshing. After it has been refreshed, the same procedure applies and once again the least activated item (which could be the same one) is refreshed and so on. This strategy was implemented by retrieving all items corresponding to all positions, then selecting the least activated.

\section{Probabilistic refreshing}

This strategy is an expansion of the previous one. Instead of putting all efforts on the least activated item, the idea is to allocate this effort over all items, depending on their activation value. Therefore, items are more likely to be refreshed if their activation value is low. The probability of refreshing an item $i$ from a set $S$ of items already encoded is:

$p(i)=\frac{1-\operatorname{activation}(i)}{\sum_{j \in S} 1-\operatorname{activation}(j)}$.

This schedule was implemented like the previous one, except that selection was random, based on the probability of each item. For instance, if there are three items with activation values of $0.5,0.2$, and 0.8 , the least activated first schedule would select the second item, whereas the current schedule would select the first one with a probability of 0.33 , the second one with a probability of 0.53 , and the third one with a probability of 0.13 .

\section{Expanded attentional focus}

The refreshing mechanism with an expanded attentional focus presented previously (Portrat \& Lemaire, 2015) was also considered. Basically, up to four items can be considered and refreshed in parallel during each refreshing episode of 80 $\mathrm{ms}$, a duration which is the same as that proposed in the default model. For instance, if items K, V, P, X, N, T have to be refreshed, the first four items $\mathrm{K}, \mathrm{V}, \mathrm{P}$, and $\mathrm{X}$ are considered first and refreshed in parallel for $80 \mathrm{~ms}$, with a strength that is shared among them. Then items $\mathrm{N}$ and $\mathrm{T}$ enter the focus of attention and are refreshed in the same way and so on.

As a matter of principle, to provide suitable candidates for this mechanism, a refreshing schedule should reproduce behavioral performance and especially the benchmark findings in immediate serial recall: primacy and recency effects. However, it is hard to make a precise prediction about which schedule is likely to reproduce the observed amplitude of primacy and recency effects. This is because it is the complex 
combination of refreshing and decay functions on hundreds of episodes within a trial that create these effects. Under these complex conditions, simulations are particularly useful. Indeed, it is not possible for us humans to predict what is going to happen to each of the letters to be maintained that have been subjected to a number of variations in their activation. Effectively, these variations depend on several factors such as the length of the list, the position of the letter in the list and the temporal characteristics of the processing task. Nevertheless, based on previous results (Portrat \& Lemaire, 2015), a simple prediction could be made: schedules that do not allocate refreshing episodes on all items of the list should not produce the recency effect when time constraints are too strong.

\section{Computational simulations}

These refreshing schedules were implemented within the computational TBRS* model mentioned previously (Oberauer \& Lewandowsky, 2011). This computational model simulates a participant performing a CST by memorizing items in a twolayer network that connects item nodes to position nodes, and recalling the items at the end of the experiment.

Figure 1 displays the two-layer structure of the model as well as an example. Basically, each item is represented by a node in the item layer, and each position is represented by a set of nodes in the position layer, such that adjacent positions share a particular proportion of nodes. Memorizing an item at a given position requires connecting the item node to the position nodes. Recalling an item at a given position involves selecting the one that is the most associated with that position. An experiment is defined by several variables: the number of items, the duration of their presentation, the number of distracting episodes between each presentation of items and the average duration of each distracting episode. The mean time taken by participants to respond to distractors is usually used as an estimation of the attentional capture induced by the processing task and is given as input to the model.

For instance, a simulation could be to memorize items B, $\mathrm{K}, \mathrm{N}, \mathrm{G}, \mathrm{V}$, each one being followed by four distracting episodes presented every 2 seconds and each one processed for an average of $600 \mathrm{~ms}$. TBRS* reproduces the four basic operations of a CST: encoding items, processing distractors, refreshing memory traces, and recalling items:

- Item encoding is performed by strengthening the links between the item node and nodes coding for the current position, for an average duration of $500 \mathrm{~ms}$.

- Distractor processing is not simulated per se, but its effect is realized by applying an exponential decay function to the item-position links during attentional capture by the distracting task.
- Refreshing occurs during the free time that follows the encoding of items or the processing of distractors. Previous positions are considered, and for each one, an item is retrieved. The retrieved item is the one whose sum of connections to the current position is maximal, after a Gaussian noise has been added to each candidate item to mimic retrieval errors. This retrieved item is then refreshed by strengthening the link to its position for an average time of $80 \mathrm{~ms}$. Because of the Gaussian noise, a wrong item can be sometimes retrieved, refreshed and then later erroneously retrieved again. In cases where all activation values are below a threshold of 0.1 , no item is retrieved and thus no refreshing occurs. It is precisely at this stage that different schedules described above are implemented.

- Recalling items follows the same mechanism as retrieval. It is performed by selecting the most activated item connected to each position node in turn.

TBRS* is based on several parameters, such as the rate of encoding, the decay rate, the average duration of encoding, and the standard deviation of the retrieval noise. To model human variability, some parameter values are merely means from which the model draws a stochastic value. The details of these parameters are described by Oberauer and Lewandowsky (2011; see also Portrat \& Lemaire, 2015).

\section{Simulation of Experimental Data 1}

\section{Experimental data}

The experimental data to which the outcomes of the various models were compared was that published by Barrouillet, Portrat, Vergauwe, et al., (2011). In their experiment, young adults performed a WM CST. They were presented with a series of seven consonants to be remembered, each consonant being followed by either four or eight black squares that were successively displayed in one of two possible locations; either the upper or the lower part of the screen. As soon as a square was displayed, participants were required to press one of two keys according to its location. The squares appeared at slow, medium, or fast paces, with one square every $1,180 \mathrm{~ms}, 980$ $\mathrm{ms}$, or $780 \mathrm{~ms}$. Experimental conditions were thus defined by these three paces and two numbers of distractors (4 or 8 ), resulting in a total of six conditions labeled $4 \mathrm{~S}, 4 \mathrm{M}, 4 \mathrm{~F}, 8 \mathrm{~S}$, $8 \mathrm{M}, 8 \mathrm{~F}$.

\section{Simulations}

The distracting tasks were not simulated per se. Only the estimated duration of their attentional capture was taken into account by the model. The experimental setup allowed 

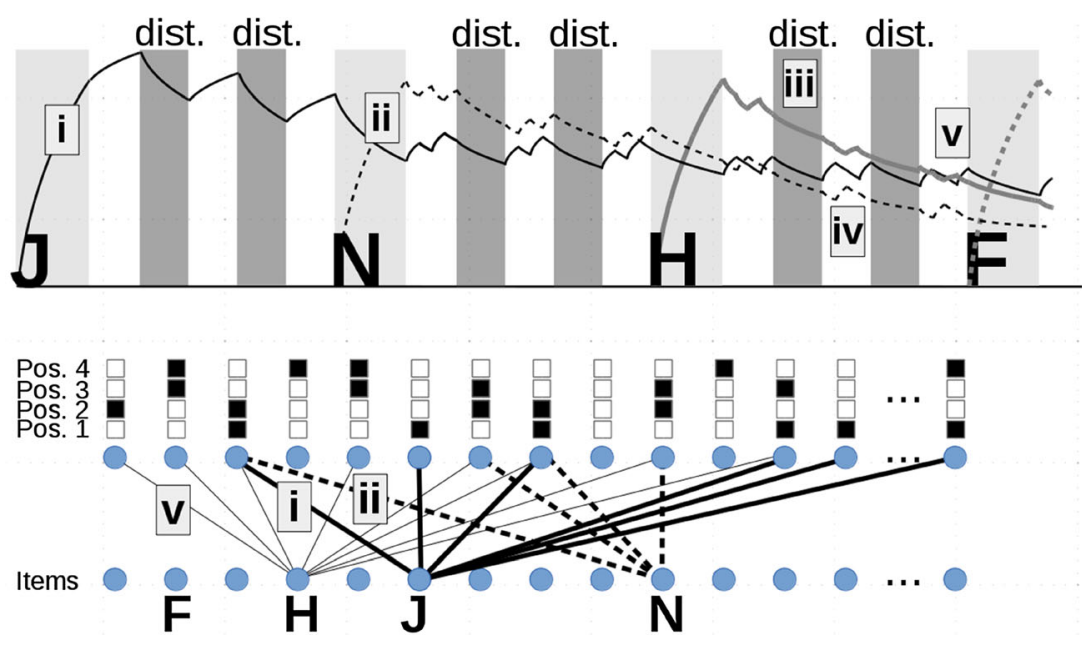

Fig. 1 Upper panel: Simulated time course of complex span task where items $J, N, H$ and $F$ are encoded (light gray areas). Two distractors are processed in between each letter presentation (dark gray areas). Free time (white areas) is used to refresh items. Curves represent total activation value of each item with respect to its position. Lower panel: Connections between position units and item units. Each item is represented by a single unit. Each position is represented by several position units. Black and white squares represent position coding, that is, Position 1 is coded by

measurement of the time between the onset of a square and the response of the participant, which was considered to be the duration of attentional capture and was used as such by the model. The average time of the conditions $4 \mathrm{~S}, 4 \mathrm{M}, 4 \mathrm{~F}, 8 \mathrm{~S}$, $8 \mathrm{M}, 8 \mathrm{~F}$ were respectively $400 \mathrm{~ms}, 388 \mathrm{~ms}, 393 \mathrm{~ms}, 395 \mathrm{~ms}$, $389 \mathrm{~ms}$, and $396 \mathrm{~ms}$. The time remaining up to the presentation of the next distractor or item was therefore considered as free time. For instance, in the $4 \mathrm{M}$ condition, a square appeared every $980 \mathrm{~ms}$. Since it took $388 \mathrm{~ms}$ to process that distractor, there were $980-388=592 \mathrm{~ms}$ left to refresh items before the next distractor or the next item materialized.

Since TBRS* is a stochastic model in which several parameters are defined as distributions rather than fixed values, many simulations had to be performed. Each simulation of each model in each condition was run 5,000 times. Table 1 displays the TBRS* parameters, which were all set to the default values that are indicated in Oberauer and Lewandowsky (2011, Table 3, p. 18).

\section{Results}

Figure 2 presents the seven models as a function of the serial position of the letters to be maintained in the series along with the recall performance of human participants observed in Barrouillet, Portrat, Vergauwe, et al., (2011). For the sake of clarity, and because the pattern of results did not change as a function of the number of distractors (Barrouillet, Portrat, Vergauwe, et al., 2011), only the three pace conditions (each resulting from averaging the performance on the four and eight distractor conditions) are displayed. The below-activation-threshold schedule
Units 3, 6, 8, 12, 13; Positions 1 and 2 share Units 3 and 8. Examples of specific processes: (i) Item $J$ is encoded and associated to all units of Position 1; (ii) Item $N$ is encoded and associated to all units of Position 2; (iii) all activation values decay during distracting task; (iv) during free time, items are retrieved and refreshed for each position in turn; (v) during the free time following the second distractor after item $H$ was presented, item $H$ was erroneously retrieved at Position 2 instead of $N$. Then, $H$ was associated to all units of Position 2

having an extra parameter, nine thresholds were tested from 0.1 to 0.9 , and only the best model is presented, corresponding to thresholds of 0.8 (refreshing duration of $80 \mathrm{~ms}$ ) and 0.5 (refreshing duration of $40 \mathrm{~ms}$ ).

Several findings can be highlighted. As already shown by Portrat and Lemaire (2015), the default model (cumulative refreshing) fails to reproduce the recency effect when the free time is short, a drawback that we also mentioned previously. When the refreshing duration $\mathrm{Tr}$ is set to $40 \mathrm{~ms}$ instead of the $80 \mathrm{~ms}$ default value, the results are slightly better, but the last items are still poorly recalled. As expected, the model that only refreshes the last encoded item is the worst one, since all items except the last are forgotten most of the time. The random model also appears to perform poorly, which is

Table 1 TBRS* default parameter values used in the present simulations

\begin{tabular}{ll}
\hline Parameter & Value \\
\hline Position marker overlap $(\mathrm{P})$ & 0.3 \\
Criterion for encoding $\left({ }^{\cdot} \tau_{\mathrm{E}}\right)$ & 0.95 \\
Processing rate $(\mathrm{R})$ & 6 \\
Standard deviation of processing rate(s) & 1 \\
Decay rate $(\mathrm{D})$ & 0.5 \\
Refreshing duration $(\mathrm{Tr})$ & $80 \mathrm{~ms}$ and $40 \mathrm{~ms}$ (see below) \\
Threshold for retrieval $(\theta)$ & 0.1 \\
Noise $(\sigma)$ & 0.02 \\
\hline
\end{tabular}

No free parameters were considered and no model fitting was therefore necessary. Two sets of simulations were performed with a refreshing duration ( $\operatorname{Tr}$ parameter) set to the default value of $80 \mathrm{~ms}$ but $40 \mathrm{~ms}$ was also used, since this is the value found by studies presented previously. 


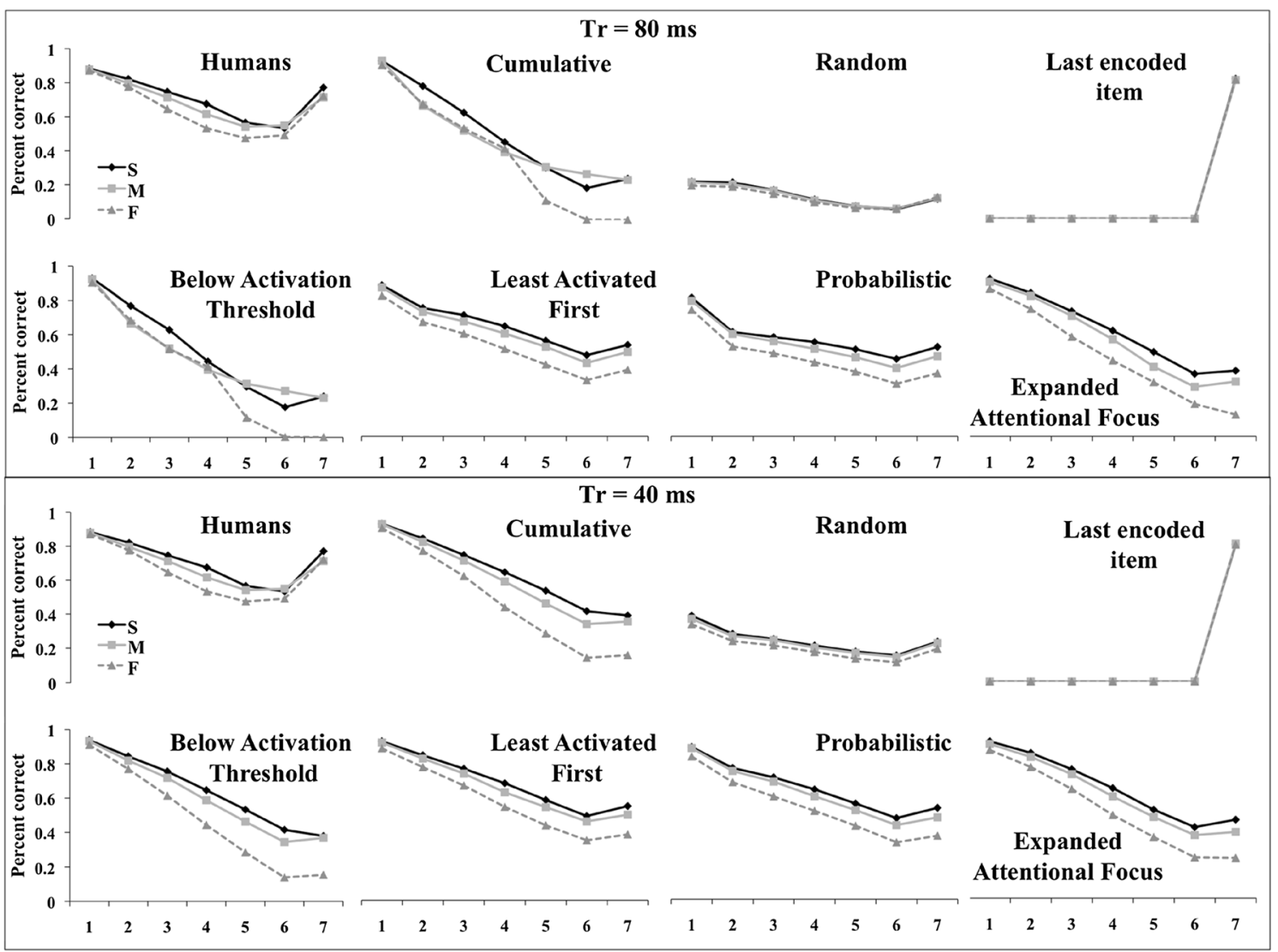

Fig. 2 Recall performance of humans as a function of the serial position of items in the series (Barrouillet, Portrat, Vergauwe, et al., 2011) and produced by the seven computational models with the refreshing duration $\operatorname{Tr}$ set at $80 \mathrm{~ms}$ (upper panel) or $40 \mathrm{~ms}$ (lower panel)

surprising because it involves a strategy that tends to consider items throughout the series in a uniform manner. This finding suggests that refreshing should not be equitable for different items. Three strategies (LAF, probabilistic, and expanded attentional focus) produced a recency effect, even at a fast pace, although not as strongly as the human recency effect.

To measure precisely the error of each strategy in predicting behavioral data, root mean square errors (RMSE) were computed. Figure 3 presents the results for each experimental condition (good models have low error values).

The LAF schedule and its probabilistic version appear to be the best in all of the six conditions. The expanded attentional focus schedule also shows very good performance. However, the default cumulative schedule produced a poor fit in the two fast pace conditions $(4 \mathrm{~F}$ and $8 \mathrm{~F})$.

The Akaike information criteria (AIC) is a more robust way of comparing models. It takes into account the goodness of fit of models with respect to experimental data and also their complexity in terms of a number of parameters. It also takes into account the data of each individual participant rather than relying on averaged data. AIC requires computing of likelihoods, which means that probability distributions should be defined. Following Lewandowsky and Farrell (2011), we computed a probability distribution over the number of correctly recalled items for each of the six conditions and each of the seven item positions over four trials, using a binomial data model of the average percentage
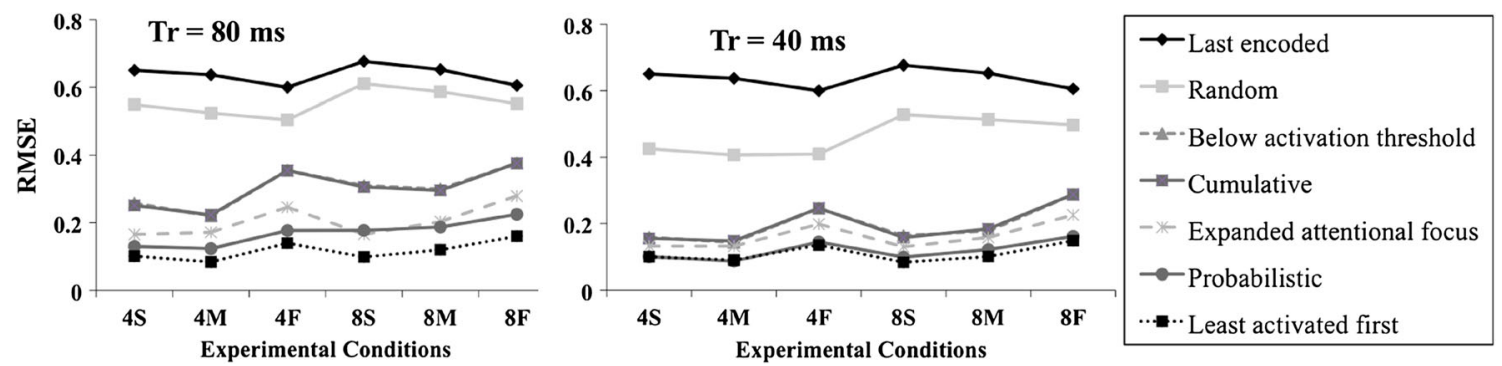

Fig. 3 RMSE between model performance and experimental data for the seven refreshing schedules and the six experimental conditions with the refreshing duration $\operatorname{Tr}$ set at $80 \mathrm{~ms}$ or $40 \mathrm{~ms}$ 
of correct responses for the model under consideration. For instance, suppose in condition $4 \mathrm{M}$ the random model has an average of $30 \%$ correct responses in Position 2. Since there are four trials per condition, the binomial formula can be used to trace the probability distribution over the five possible outcomes: $0,1,2,3$, or 4 correct recalls in Position 2 (see Figure 4).

All experimental data are then considered individually to compute individual likelihoods for each condition and each position. To continue our example, if a participant correctly remembered the second item in condition $4 \mathrm{M}$ three times, according to the probability distribution this individual likelihood will be 0.0756 . This is not a good support for the random model. Participants recalling only one item out of four would lead to an individual likelihood of 0.41 , which would be a much better support for that model. The likelihood of each model is simply the product of all individual likelihoods, for all participants, all conditions and all positions (see Portrat et al., 2016, for a similar evaluation method).

AIC is defined as $-2 \log (L)+2 k, L$ being the likelihood and $k$ being the number of free parameters of the model. Obviously, the lower AIC, the better the model. All our models use eight free parameters except the below activation threshold schedule where the threshold is an extra parameter and the expanded attentional focus schedule for which the size of the attentional focus can also be considered as an additional parameter, although there is experimental evidence for the reliability of this value. Since raw AIC differences are not easy to interpret, we computed AIC weights that are viewed as the probability of each model being the best one (Wagenmakers \& Farrell, 2004). Table 2 gives the AIC values and their weights for each of the seven models and the two refreshing duration (Tr) values.

Once again, the LAF schedule appears to be the closest to the experimental data. When the duration of individual refreshing is set to $40 \mathrm{~ms}$, differences between models are narrowed since a shorter duration gives more opportunity to refresh the last items. The three best schedules are still the same. The LAF schedules, either in their raw or probabilistic versions, outperform the other

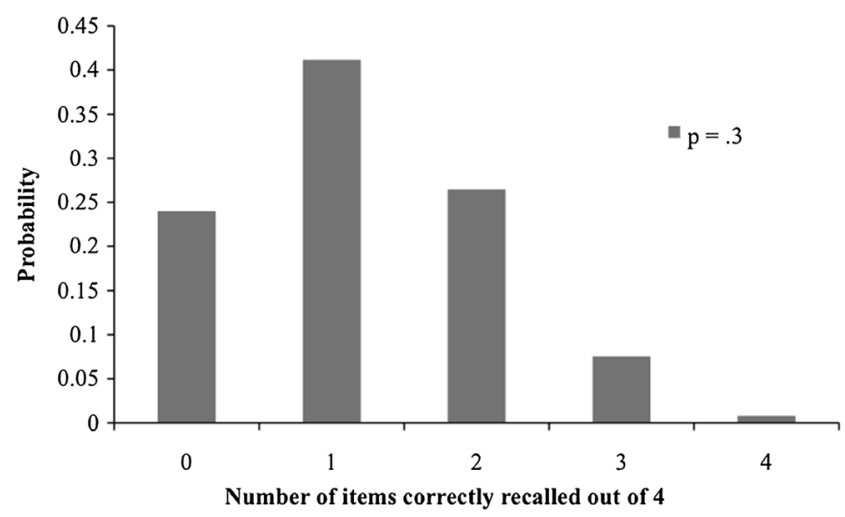

Fig. 4 Probability of correctly recalling $N$ out of four items when the percentage of correct responses at a given position is $p=30 \%$ ones. To refresh memory items, participants tend to focus their attention on items that are the most likely to be forgotten. However, the expanded attentional focus schedule appears to be a possible competitor to LAF schedules.

\section{Simulations of experimental data 2}

\section{Experimental data}

To check the robustness of previous results, new simulations were performed in another experiment (Barrouillet, Portrat, \& Camos, 2011), with a different response mode for the distracting tasks. In the previous experiment, where participants had to respond to the distractor by pressing a key, they could have relied on articulatory rehearsal, instead of attentional refreshing, as a way to maintain items. Nonetheless, it is worth noting that it would have been quite difficult to rehearse items in fast conditions. Indeed, the time pressure of the processing task in such conditions would have seriously impeded the mental articulation of lists containing up to seven letters. However, to definitely rule out this argument, the current set of data comes from an experiment in which participants had to produce oral responses to the distracting tasks, since these prevent an articulatory rehearsal strategy. In these experiments, distracting episodes were Stroop-like tasks; participants had to respond out loud by either naming the color in which a word appeared on screen (color Stroop task) or evaluating the number of a small set of stimuli (numerical Stroop task). With such verbal distracting tasks, we can be very confident that the recall performance collected depended primarily on the refreshing mechanism that is under study here.

Participants were required to perform a CST as previously, but the design was slightly different. They followed a span procedure in which they had to recall lists of three, four, five, and six items. After each item to be maintained, there were eight distractors to process according to either a color or a

Table 2 AIC and weights for each of the seven models simulating Experimental Data 1, in ascending order, for two different refreshing durations $(\mathrm{Tr})$

\begin{tabular}{lllllll}
\hline \multirow{2}{*}{ Model } & \multicolumn{2}{l}{$\operatorname{Tr}=80 \mathrm{~ms}$} & & & $\operatorname{Tr}=40 \mathrm{~ms}$ \\
\cline { 2 - 3 } \cline { 6 - 7 } \cline { 5 - 6 } & AIC & Weight & & AIC & Weight \\
\hline Least activated first & 3830.7 & 1 & & 3796.7 & 1 \\
Probabilistic & 4177.8 & $10^{-76}$ & & 3838.6 & $10^{-10}$ \\
Expanded attentional focus & 4577.3 & 0 & & 4140.5 & $10^{-75}$ \\
Below activation threshold & 8480.4 & 0 & & 4522.8 & 0 \\
Cumulative (default) & 9200.7 & 0 & & 4523.5 & 0 \\
Random & 12206.0 & 0 & & 8752.4 & 0 \\
Last encoded & 52047.3 & 0 & & 52043.3 & 0 \\
\hline
\end{tabular}


numerical Stroop-like task. It is not important to specify in detail the nature of these distracting tasks, since they are not simulated per se (see Barrouillet, Portrat, \& Camos, 2011, pp. 180, 191 for details).

\section{Simulations}

As explained above, only the duration of the attentional capture imposed by the processing tasks is taken into account by the model. The average duration of processing activities for the color Stroop task was $668 \mathrm{~ms}$ in the color-word condition and $617 \mathrm{~ms}$ in the neutral-word condition. For the numerical Stroop task, the mean response times were $603 \mathrm{~ms}$ and $545 \mathrm{~ms}$ for the interfering and the neutral condition, respectively. As previously, all parameters of TBRS* were set to their default values and the two refreshing durations of $80 \mathrm{~ms}$ and $40 \mathrm{~ms}$ were used. The number of runs was set to 5,000 per condition.

\section{Results}

Simulation results were assessed according to the two methods presented above. First, serial position curves and RMSE were used to evaluate the ability of the model to replicate the average recall performance of humans. Then, AIC measurements provided even more valuable data to compare models by considering individual human behaviors.

Figure 5 displays the serial position curves of humans and the three main models under consideration. When the refreshing rate is set to the default value of $80 \mathrm{~ms}$, the cumulative schedule is still unable to account for the recency effect in both color Stroop and numerical Stroop tasks. The expanded attentional focus schedule seems to perform slightly better than the least-activated-first (LAF) in reproducing human serial position curves, which leads to a lower RMSE.

When the refreshing rate is set to $40 \mathrm{~ms}$, all schedules behave more or less in similar ways. For the color Stroop task, averaged RMSE are 0.09 (cumulative), 0.09 (expanded attentional focus), 0.11 (LAF), whereas for the numerical Stroop task, averaged RMSE are 0.07 (expanded attentional focus), 0.08 (LAF), 0.08 (cumulative).

A raw comparison of averaged human data is therefore not very informative. The cumulative schedule appears to be highly dependent on the refreshing rate, which is not the case for the other schedules that are quite robust. Taking into account individual experimental data is then necessary. Table 3 shows the AIC values for each experiment, in ascending order. Likelihood values were computed as previously, except that the number of trials in each condition was three instead of four.

The ranks of each schedule are almost the same in the different sets of data, which indicates a high level of confidence in the method. The results of the second simulation are in line with the previous one, except that the expanded attentional focus schedule then appears to be the best model, LAF being the second best one.

\section{Simulations of artificial data}

We then performed another set of simulations to check whether the LAF schedule that is proposed in this paper could have affected the general behavior of the TBRS* model and, in particular, its ability to reproduce some of the classical WM effects found in the literature. To that end, we simulated the hypothetical experiment presented in Oberauer and Lewandowsky (2011), in which the number of distractors inbetween each item presented were manipulated $(0,1,4$, or 8$)$, along with the duration of the attentional capture of distractors (300 ms, $500 \mathrm{~ms}$ or $700 \mathrm{~ms}$ ) and the free time following each distractor (0 ms, $100 \mathrm{~ms}, 600 \mathrm{~ms}, 1200 \mathrm{~ms}$, or $2000 \mathrm{~ms}$ ). Figure 6 shows the model performance as a function of the free time and the operation duration for 8 operations per burst, exactly as was done with the cumulative schedule by Oberauer and Lewandowsky (2011).

It appears that the LAF schedule is able to reproduce the cognitive load effect for the two refreshing durations. When there is more free time after a given processing duration performance increases and, inversely, when the attentional capture by the distractor increases for a given free time, performance decreases. The new schedule we propose is therefore able to reproduce the benchmark findings that support the TBRS verbal theory. It is worth noting that the expanded attentional focus schedule is also able to reproduce the cognitive load effect in this fictional experiment.

\section{Discussion}

This study aimed at an in-depth investigation of a maintenance mechanism, attentional refreshing, that is crucial in WM. It is important to understand refreshing in detail, because it is an elementary cognitive mechanism, which constitutes the keystone of the decay-based view of WM. However, specifying the time course of this mechanism when taking place in WM is difficult. Indeed, it is a very rapid mechanism that contributes to recall performance throughout the task, but which also interacts with other mechanisms, phenomena or variables (e.g., time-based decay, rehearsal, quantity of memory items), these interactions still being underspecified. In such cases, where researchers cannot fully specify how the elementary mechanisms suggested by a theory combine because of the complexity of the problem at hand, a powerful approach is to implement these mechanisms in a computer and observe how they combine. We therefore investigated different refreshing schedules using a computational modeling approach within TBRS* (Oberauer \& Lewandowsky, 2011) of the TBRS 


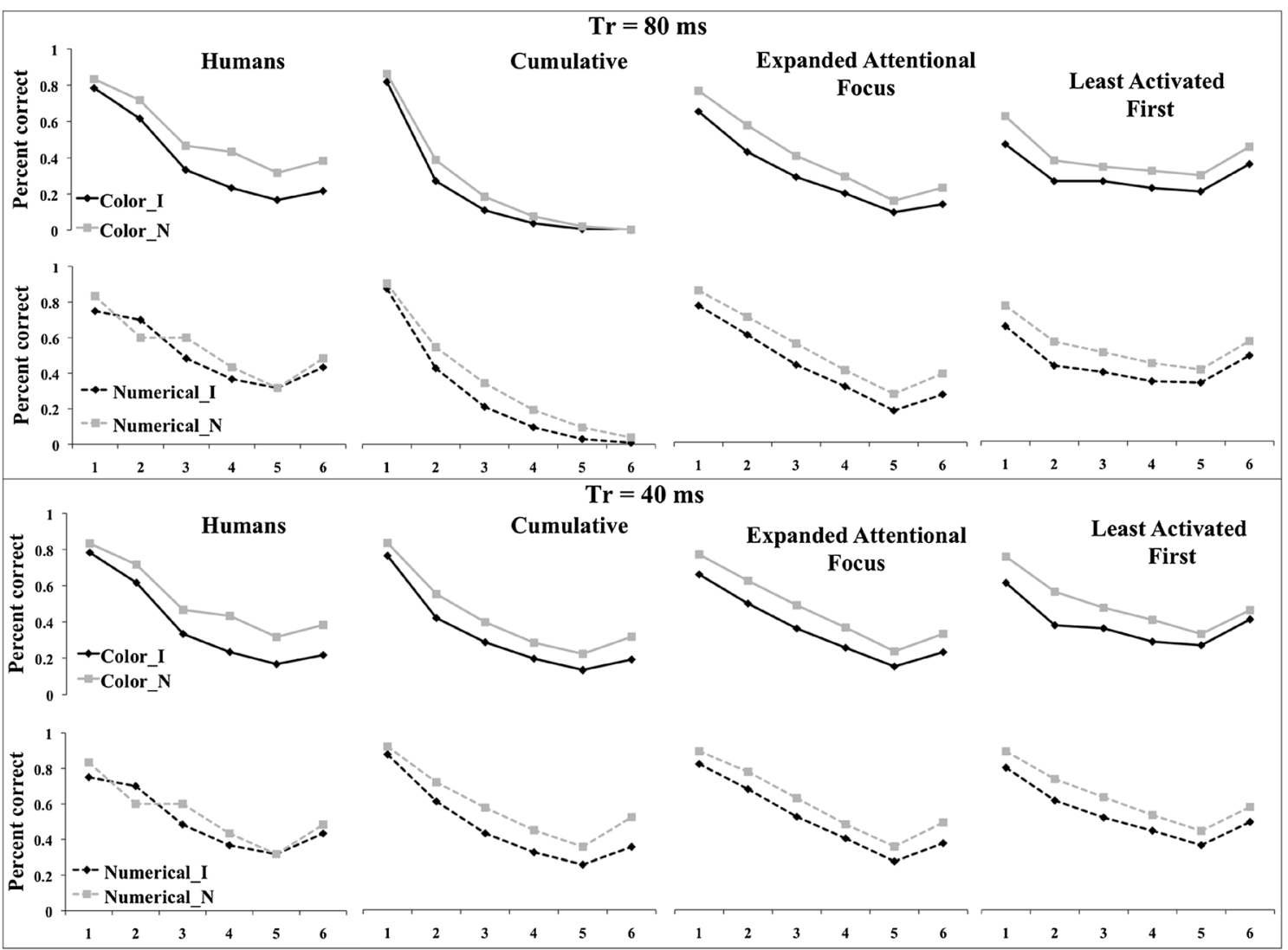

Fig. 5 Recall performance as a function of the serial position of items in the series observed by humans (Barrouillet, Portrat, \& Camos, 2011) and expanded attentional focus, and least-activated-first refreshing, with the refreshing duration $\mathrm{Tr}$ set at $80 \mathrm{~ms}$ (upper panel) and $40 \mathrm{~ms}$ (lower panel) produced by the different models including cumulative refreshing,

verbal theory (Barrouillet, Bernardin, Portrat, Vergauwe, \& Camos, 2007).

Before going further, it is worth noting that the attentional refreshing maintenance mechanism actually has two meanings in the literature. First of all, it refers to the reactivation of a single item during $40 \mathrm{~ms}$ or $80 \mathrm{~ms}$ (Jarrold et al., 2011; Johnson et al., 2004; Johnson et al., 2005; Johnson et al., 2002; Oberauer \&
Lewandowsky, 2011; Vergauwe et al., 2014; Vergauwe \& Cowan, 2015). We proposed elsewhere to call it "atomic refreshing" (Portrat \& Lemaire, 2015). However, in the context of WM and complex span tasks, refreshing also refers to a broader general procedure aiming at maintaining memory traces with the goal of recalling latter items. Indeed, following Barrouillet, Camos, and colleagues, refreshing is viewed as a

Table 3 AIC (rank) and weights (Wgt) for each of the seven models using the color-Stroop and numerical-Stroop data, in ascending order, for refreshing duration values (Tr) of $80 \mathrm{~ms}$ and $40 \mathrm{~ms}$

\begin{tabular}{|c|c|c|c|c|c|c|c|c|}
\hline \multirow[b]{3}{*}{ Models } & \multicolumn{4}{|l|}{$\operatorname{Tr}=80 \mathrm{~ms}$} & \multicolumn{4}{|l|}{$\operatorname{Tr}=40 \mathrm{~ms}$} \\
\hline & \multicolumn{2}{|c|}{ Color Stroop } & \multicolumn{2}{|c|}{ Numerical Stroop } & \multicolumn{2}{|c|}{ Color Stroop } & \multicolumn{2}{|c|}{ Numerical Stroop } \\
\hline & AIC & Wgt & AIC & Wgt & AIC & Wgt & AIC & Wgt \\
\hline Expanded attentional focus & 1849 (1) & 1 & 1779 (1) & .999 & $1847(1)$ & 1 & $1774(1)$ & .989 \\
\hline Least activated first & $1952(2)$ & $10^{-23}$ & $1800(2)$ & $10^{-5}$ & $1889(2)$ & $10^{-10}$ & $1783(2)$ & .011 \\
\hline Probabilistic & $2111(3)$ & 0 & $1985(3)$ & $10^{-45}$ & $1924(4)$ & $10^{-17}$ & $1799(3)$ & $10^{-6}$ \\
\hline Below activation threshold & $2782(5)$ & 0 & $2552(5)$ & 0 & $1893(3)$ & $10^{-10}$ & $1818(5)$ & $10^{-10}$ \\
\hline Cumulative (default) & 2814 (4) & 0 & $2519(4)$ & 0 & $2096(5)$ & 0 & $1818(4)$ & $10^{-10}$ \\
\hline Random & $3109(6)$ & 0 & $3419(6)$ & 0 & $2603(6)$ & 0 & $2649(6)$ & 0 \\
\hline Last encoded & $17982(7)$ & 0 & $20701(7)$ & 0 & $17962(7)$ & 0 & $20694(7)$ & 0 \\
\hline
\end{tabular}



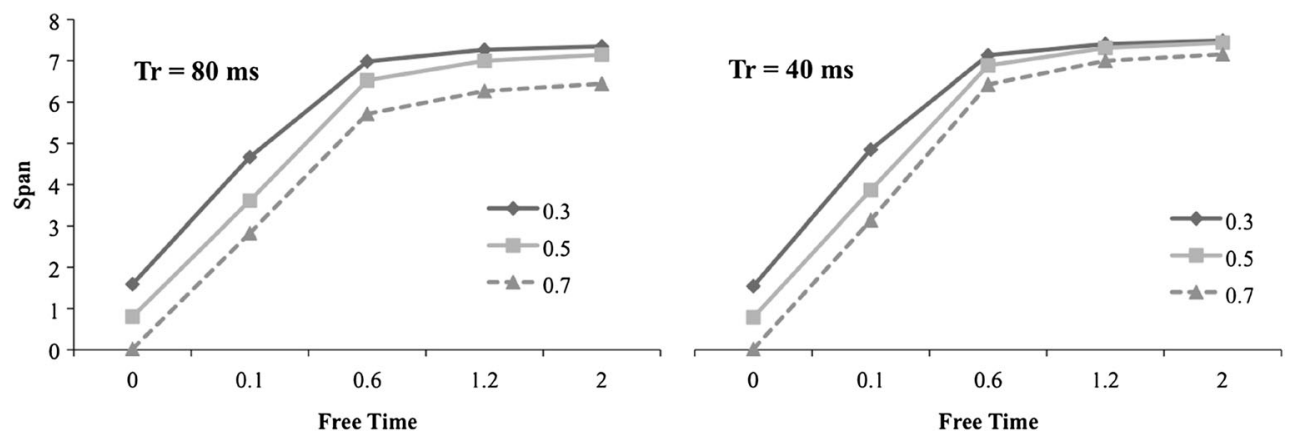

Fig. 6 Model performance for the least-activated-first schedule as a function of the free time (in s) and operation duration $(0.3 \mathrm{~s} ; 0.5 \mathrm{~s}$ and $0.7 \mathrm{~s}$; for eight operations per burst) with the refreshing duration Tr set at $80 \mathrm{~ms}$ and $40 \mathrm{~ms}$

strategy that is chosen from among other possibilities such as verbal rehearsal (Camos et al., 2011) and that could explain why individuals differ in WM capacity both developmentally (Camos \& Barrouillet, 2011; Gaillard, Barrouillet, Jarrold, \& Camos, 2011) and in adulthood (Barrouillet, Lépine, \& Camos, 2008). The present study goes further, by proposing that this global strategic refreshing is composed of two subprocesses: performing "atomic refreshing" operations but, first, selecting which item to reactivate at a given time. The subprocess that selects the item to be reactivated is the one studied in the present paper. Although the global refreshing strategy, as well as its atomic refreshing component, have an attentional cost, the selection of the item to be reactivated could be rather automatic or even automated with experience and development.

\section{Refreshing schedules}

The present study calls into question the classical cumulative conception of refreshing which, akin to the well-known articulatory rehearsal, consists of a loop that would operate in forward order from the beginning until the end of the series (e.g., Jarrold et al., 2011; Loaiza \& McCabe, 2012; McCabe, 2008; Vergauwe et al., 2014). That cumulative schedule does not appear to accurately reproduce the experimental data. A schedule based on an expanded attentional focus, where up to four items can be refreshed in parallel, accommodated the experimental data better, replicating previous findings (Portrat \& Lemaire, 2015). However, the fact that several items could be refreshed at the same time is under debate. Indeed, in general theories of cognition, it has been proposed that central cognition is serial (Anderson, 1993; Newell, 1990). For example, in the ACT-R model (Anderson \& Lebiere, 1998) the atomic steps of cognition consist in firing productions rules that are assumed to be fired only one at a time. According to the concept of central bottleneck (Pashler, 1998), central processes like response selection can only take place one at a time and the following processes are thus postponed. Moreover, regarding attentional refreshing, different findings suggested that the size of the focus of attention is limited to only one element (Garavan, 1998; McElree \& Dosher, 1989; Oberauer, 2002, 2005).
Hence, when restricted to the more consensual sequential models, our simulations showed that one schedule outperforms all the others across three simulation sets. When the least-activated item is refreshed first, the model reproduces quite well the experimental data obtained by adults over three different experiments and is also able to produce the benchmark findings that support the TBRS verbal theory. Moreover, this new schedule is still compatible with the recent estimates of about 40 to $50 \mathrm{~ms}$ to refresh a single item (Jarrold et al., 2011; Vergauwe et al., 2014; Vergauwe \& Cowan, 2015), although being not as dependent on that specific duration as the classical cumulative schedule. From a computational perspective, it is worth noting that this new schedule does not require any additional parameters and thus did not require any parameter fitting. This is an important result because it is easier to fit data well when a new degree of freedom is allowed.

One could wonder how the memory trace with the lowest activation is selected. Our implementation on a sequential computer processor consists of computing activation values of each item in turn and keeping the minimum value. This has no resemblance to the way it would happen in a distributed architecture like the human brain. The simulation of this sequential mechanism implemented for the selection of the next to be refreshed item did not have any cost. Hence, any other option, sequential, parallel or brain-like, would have produced the same output. A distinction should be made between a cognitive process and its implementation (i.e., between the processes operating in a model and the way they become operational). Marr (1982) proposed that any system, natural or artificial, designed to perform an information processing task can be described at three different levels: the computational level (what the system does), the algorithmic level (how the system does it) and the implementation level (on which physical substrate). The LAF schedule was described in this study at the computational level, without any particular assumption as to how the appropriate item is selected for the next refreshing step. Actually, there are different ways to perform that schedule (i.e., to describe it at the algorithmic level). Oberauer and Lewandowsky (2011, p. 33) believe that such a mechanism would imply that an omniscient homunculus performs the selection. This question applies to all the possible 
scheduling strategies: even the cumulative schedule requires a mechanism to manage the move to the next position. This issue is shared by most of the current WM models where the control of WM resource assignments across different cognitive activities remains unresolved (Baddeley, 2000; Cowan, 2005; Barrouillet, Bernardin, \& Camos, 2004; Engle, Tuholski, Laughlin, \& Conway, 1999).

\section{Primacy and recency effects}

The quality of a complex model is often assessed with respect to its ability to explain strong effects found in behavioral data. Primacy and recency effects are examples of such widely observed markers, and it is worth studying to what extent they depend on refreshing schedules. It is generally considered that the primacy effect comes from the fact that the first items are strongly encoded and refreshed, because they were alone at the beginning of the trial, whereas the recency effect occurs because last items did not have time to be forgotten. However, the picture is probably more complicated than this. Primacy and recency effects are strong markers that models should reproduce, but their amplitudes are not well-specified yet, because they depend on interacting factors throughout a trial: notably time-based decay and item reactivation.

At first sight, except for the last-encoded schedule, it seems that all of our simulations reproduced the characteristic U-shape which could indicate that primacy and recency effects do not depend on the refreshing schedules. However, the amplitude of these effects clearly depends on the schedule and thus on the distribution of refreshing and decay episodes throughout the trial. For instance, the cumulative and below-activationthreshold produced a recency effect only at a slow pace, and the primacy effect is very thin when considering the random schedule. Another argument in favor of a relationship between the recency effect and refreshing is that this effect disappears when the last items are not refreshed. Indeed, at a fast pace, simulations of the cumulative schedule show that the last item is not refreshed at all. Although it was recently encoded, the last memory trace cannot resist decay during the last refreshing episode and during the recall step. As soon as the refreshing schedule allows it, the last item is refreshed during the last distracting episode and the recency effect is reproduced. However, the poor result of the random schedule, which operates in a uniform manner, showed that the refreshing process is probably not fully equitable. Consequently, refreshing probably contributes to the recency effect, although less strongly than to the primacy effect.

\section{Refreshing within the general cognitive system}

As mentioned above, one would argue that a homunculus is needed to select the least activated item in particular. However, we believe that several arguments from various fields of cognitive psychology could discard this argument. First of all, Vandierendonck (2016) recently proposed a WM system with a distributed executive control that functions without any supra control such as a homunculus. According to this framework, the executive control that is required to select an action is mediated by the goal that is pursued in the given cognitive task that requires this action. The homunculus (referred as the central executive in Baddeley's model) is deleted and replaced by three interacting components: acquired procedural rules are executed through an engine if they match the sum of WM contents, including the task set maintained by an executive memory. This proposal resembles the procedural WM in Oberauer's model $(2009,2010)$.

If we apply this theoretical model to the CST used in the present study, several concurrent goal representations are maintained during task execution. These goals can be described as follows: encode some items, try to maintain them as best possible and thus refresh them and process some other items (e.g., judge the location of a square). According to Vandierendonck's model, the specific rules corresponding to the processing goal are automatically triggered when they are primed by the stimulus presented on the screen. For example, when a square is presented, given that the square constitutes an environmental change, the attention is captured, leading to the start of the processing goal (e.g., location-judgment goal) and to the run of the corresponding task. A process of inhibition prevents the activation of the memorization task, to keep the processing task set dominant. Once the processing task is over, the refreshing task set becomes dominant. Items are thus refreshed until the next processing event. The rest of the trial consists of switching back and forth between refreshing and processing tasks. The refreshing mechanism would be triggered when memory traces are close to being lost because procedural rules are instated as a function of the WM content and because the main goal is to maintain items at their best despite a concurrent distracting goal. Hence, when refreshing is possible, that is when attention is not captured by external events, it would focus on the precise memory item that is in need of refreshing (i.e., the least activated one). As we will discuss later, this specific schedule is most likely to be activated when the task featured (e.g., the pace of the processing activity) imposes a high maintenance efficacy.

Secondly, thanks to the self-organization of our cognitive system (Perruchet \& Vinter, 2002), it is probable that the next item to be refreshed depends on its level of activation, and it can be predicted from elementary learning processes. Since the rhythm of alternating between distractors and refreshing is constant within a trial and the number of items to refresh is also known, the cognitive system can predict when to refresh a specific item, given its previous activation value, without having to constantly control current activation values. Let us draw an analogy with a chef who is cooking different sauces at the same time. He has to control several saucepans and has to mix 
each one regularly to avoid the contents from sticking. The cumulative schedule would be to mix all the saucepans for a short time in order. The LAF schedule would be to perform an action on the saucepan whose contents are the most likely to stick. This can be done in two different ways. The first consists of quickly scanning all the saucepans' contents and selecting the one most likely to stick, then mixing that one before starting again the scan of all saucepans. The second would be to keep in mind the time at which a check is necessary for each saucepan. Since the initial state of each saucepan is known and the process of "decay" of the content is also known from experience, the chef can predict the state of each saucepan without having to check them all visually. For instance, he could know that a particular saucepan does not have to be checked until, for example, every 2 minutes. Returning to WM, not all item activation values have to be continuously kept in mind to be able to select which item needs attention and refreshing. Given that phenomenal experience is selforganizing (Perruchet \& Vinter, 2002), the process and rate of decay of memory traces could have been learned thanks to the many memorization situations experienced by an adult throughout his life. This knowledge could thus allow the cognitive system to select precisely the items that are in need of refreshing at a given time.

As a consequence, it is not necessary to assume the existence of a homunculus that manages refreshing strategies. We also showed that the LAF schedule could be performed by the cognitive system at the algorithmic level, without requiring a constant scan of all items. As suggested earlier, it is possible that when the cognitive load is not high, the cumulative schedule works well, because under such conditions sophisticated scheduling is not that important. However, when the time pressure is high, an ecological and efficient scheduling strategy becomes crucial and priorities have to be set. Following Koriat (2005) and Vandierendonck (2016), it may be considered that the different schedules could be encoded in long-term memory as procedures to achieve a goal (the goal being successful recall). Initially, the use of such a procedure would require a lot of control, but with practice, the method would become more automatic. It could also be possible that the best two schedules (LAF and expanded attentional focus) coexist and can be invoked in function of the characteristics of the task. The precise conditions that would favor one schedule or the other still need to be delineated. Moreover, it is even possible that both schedules combine and operate at the same time for a given set of memoranda. For instance, an expanded attentional focus schedule could reactivate the $\mathrm{n}$ least-activated items.

To sum up, humans may dispose of several refreshing schedules that are differentially started depending on intrinsic (e.g., individual attentional capacity, Engle \& Kane, 2004; level of motivation and metacognition, Carretti, Borella, Zavagnin, \& De Beni, 2011) or external (e.g., instruction, Campoy \& Baddeley, 2008; concurrent processing task,
Camos, Mora, \& Oberauer, 2011; acronyms in the memoranda, Portrat et al., 2016) factors. The next step will be to generalize or to specify the specific conditions under which the LAF schedule and the expanded attentional focus take place. Computational modeling seems to be a fruitful method for investigating such a rapid cognitive mechanism.

\section{References}

Altmann, E. M., \& Schunn, C. D. (2012). Decay versus interference: A new look of at an old interaction. Psychological Science, 23, 14351437.

Anderson, J. (1993). Rules of the mind. Hillsdale, NJ: Erlbaum.

Anderson, J. R., \& Lebiere, C. (1998). The atomic components of thought. Mahwah, NJ: Erlbaum.

Anderson, J. R., Reder, L. M., \& Lebiere, C. (1996). Working memory: Activation limitations on retrieval. Cognitive Psychology, 30, 221256.

Baddeley, A. D. (1986). Working memory. Oxford, UK: Clarendon Press.

Baddeley, A. D. (2000). The episodic buffer: A new component of working memory? Trends in Cognitive Science, 4, 417-423.

Baddeley, A. D., \& Hitch, G. (1974). Working memory. In G. H. Bower (Ed.), The psychology of learning and motivation: Advances in research and theory (Vol. 8, pp. 47-89). New York, NY: Academic Press.

Barrouillet, P., Bernardin, S., Portrat, S., Vergauwe, E., \& Camos, V. (2007). Time and cognitive load in working memory. Journal of Experimental Psychology: Learning, Memory, and Cognition, 33, 570-585.

Barrouillet, P., Bernardin, S., \& Camos, V. (2004). Time constraints and resource sharing in adults' working memory spans. Journal of Experimental Psychology: General, 133, 83-100.

Barrouillet, P., \& Camos, V. (2015). Working memory: Loss and reconstruction. Hove, UK: Psychology Press.

Barrouillet, P., Lépine, R., \& Camos, V. (2008). Is the influence of working memory capacity on high level cognition mediated by complexity or resource-dependent elementary processes? Psychonomic Bulletin and Review, 15, 528-534.

Barrouillet, P., Portrat, S., Vergauwe, E., Diependaele, K., \& Camos, V. (2011). Further evidence for temporal decay in working memory. Journal of Experimental Psychology: Learning, Memory, and Cognition, 37, 1302-1317.

Barrouillet, P., Portrat, S., \& Camos, V. (2011). On the law relating processing to storage in working memory. Psychological Review, 118, 175-92.

Camos, V., \& Barrouillet, P. (2011). Factors of working memory development: The time-based resource-sharing approach. In P. Barrouillet \& V. Gaillard (Eds.), Cognitive development and working memory: From neo-Piagetian to cognitive approaches (pp. 151-176). Hove, UK: Psychology Press.

Camos, V., \& Barrouillet, P. (2014). Attentional and non-attentional systems in the maintenance of verbal information in working memory: The executive and phonological loops. Frontiers in Human Neuroscience, 8, 1-11.

Camos, V., Lagner, P., \& Barrouillet, P. (2009). Two maintenance mechanisms of verbal information in working memory. Journal of Memory and Language, 61, 457-469.

Camos, V., Mora, G., \& Barrouillet, P. (2013). Phonological similarity effect in complex span task. Quarterly Journal of Experimental Psychology, 66(10), 1927-1950. 
Camos, V., Mora, G., \& Oberauer, K. (2011). Adaptive choice between articulatory rehearsal and attentional refreshing in verbal working memory. Memory \& Cognition, 39, 231-244.

Camos, V., \& Portrat, S. (2015). The impact of cognitive load on delayed recall. Psychonomic Bulletin and Review, 22, 1029-1034.

Campoy, G., \& Baddeley, A. D. (2008). Phonological and semantic strategies in immediate serial recall. Memory, 16, 329-340.

Carretti, B., Borella, E., Zavagnin, M., \& De Beni, R. (2011). Impact of metacognition and motivation on the efficacy of strategic memory training in older adults: Analysis of specific, transfer and maintenance effects. Archives of Gerontology and Geriatrics, 52, e192e197.

Cowan, N. (1999). An embedded-process model of working memory. In A. Miyake \& P. Shah (Eds.), Models of working memory: Mechanisms of active maintenance and executive control (pp. 62101). Cambridge, UK: Cambridge University Press.

Cowan, N. (2005). Working memory capacity. Hove, UK: Psychology Press.

Cowan, N., Saults, J. S., \& Elliott, E. M. (2002). The search for what is fundamental in the development of working memory. Advances in Child Development and Behavior, 29, 1-49.

Craik, F. I. M., \& Lockhart, R. S. (1972). Levels of processing: A framework for memory research. Journal of Verbal Learning and Verbal Behavior, 11, 671-684

Engle, R. W., \& Kane, M. J. (2004). Executive attention, working memory capacity, and a two-factor theory of cognitive control. In The psychology of learning and motivation (Vol. 44, pp. 145-199). New York, NY: Elsevier.

Engle, R. W., Tuholski, S. W., Laughlin, J. E., \& Conway, A. R. A. (1999). Working memory, short-term memory and general fluid intelligence: A latent variable approach. Journal of Experimental Psychology: General, 128, 309-331.

Gaillard, V., Barrouillet, P., Jarrold, C., \& Camos, V. (2011). Developmental differences in working memory: Where do they come from? Journal of Experimental Child Psychology, 110, 469479.

Garavan, H. (1998). Serial attention within working memory. Memory \& Cognition, 26, 263-276.

Hoareau, V., Lemaire, B., Portrat, S., \& Plancher, G. (2016). Reconciling two computational models of working memory in aging. Topics in Cognitive Science, 8, 264-278.

Hudjetz, A., \& Oberauer, K. (2007). The effects of processing time and processing rate on forgetting in working memory: Testing four models of the complex span paradigm. Memory \& Cognition, 35, $1675-1684$

Jarrold, C., Tam, H., Baddeley, A. D., \& Harvey, C. E. (2011). How does processing affect storage in working memory tasks? Evidence for both domain-general and domain-specific effects. Journal of Experimental Psychology: Learning, Memory \& Cognition, 37, 688-705.

Johnson, M. K. (1992). MEM: Mechanisms of recollection. Journal of Cognitive Neuroscience, 4, 268-280.

Johnson, M. K., Mitchell, K. J., Raye, C. L., \& Greene, E. J. (2004). An age-related deficit in prefrontal cortical function associated with refreshing information. Psychological Science, 15, 127-32.

Johnson, M. K., Raye, C. L., Mitchell, K. J., Greene, E. J., Cunningham, W. A., \& Sanislow, C. A. (2005). Using fMRI to investigate a component process of reflection: Prefrontal correlates of refreshing a just-activated representation. Cognitive, Affective, \& Behavioral Neuroscience, 5, 339-361.

Johnson, M. K., Reeder, J. A., Raye, C. L., \& Mitchell, K. J. (2002). Second thoughts versus second looks: An age-related deficit in reflectively refreshing just-activated information. Psychological Science, 13, 64-67.

Koriat, A. (2005). Are we frightened because we run away? Some evidence from metacognitive feelings. In B. Uttl, N. Ohta, \& A. L.
Siegenthaler (Eds.), Memory and emotions: Interdisciplinary perspectives. Malden, MA: Blackwell.

Lewandowsky, S., \& Farrell, S. (2011). Computational modeling in cognition: Principles and practice. Thousand Oaks, CA: SAGE.

Loaiza, V. M., \& McCabe, D. P. (2012). Temporal contextual processing in working memory: Evidence from delayed cued recall and delayed free recall tests. Memory \& Cognition, 40, 191-203.

Marr, D. (1982). Vision: A computational investigation into the human representation and processing of visual information. New York, NY: W. H. Freeman.

McCabe, D. P. (2008). The role of covert retrieval in working memory span tasks: Evidence from delayed recall tests. Journal of Memory and Language, 58, 480-494.

McElree, B., \& Dosher, B. A. (1989). Serial position and set size in shortterm memory: Time course of recognition. Journal of Experimental Psychology: General, 18, 346-373.

Mora, G., \& Camos, V. (2013). Two systems of maintenance in verbal working memory: Evidence from the word length effect. PLoS ONE, 7(8), 1-8.

Newell, A. (1990). Unified theories of cognition. Cambridge, MA: Harvard University Press.

Oberauer, K. (2002). Access to information in working memory: Exploring the focus of attention. Journal of Experimental Psychology: Learning, Memory, and Cognition, 28, 411-421.

Oberauer, K. (2005). Control of the contents of working memory $-\mathrm{A}$ comparison of two paradigms and two age groups. Journal of Experimental Psychology: Learning, Memory, and Cognition, 31, 714-728.

Oberauer, K. (2009). Design for a working memory. Psychology of Learning and Motivation, 51, 45-100.

Oberauer, K. (2010). Declarative and procedural working memory: Common principles, common capacity limits? Psychologica Belgica, 50, 277-308.

Oberauer, K., \& Lewandowsky, S. (2011). Modeling working memory: A computational implementation of the time-based resource-sharing theory. Psychonomic Bulletin \& Review, 18, 10-45.

Oberauer, K., Lewandowsky, S., Farrell, S., Jarrold, C., \& Greaves, M. (2012). Modeling working memory: An interference model of complex span. Psychonomic Bulletin \& Review, 19, 779-819.

Pashler, H. E. (1998). The psychology of attention. Cambridge, MA: MIT Press.

Perruchet, P., \& Vinter, A. (2002). The self-organizing consciousness. Behavioral and Brain Sciences, 25, 297-388.

Portrat, S., Guida, A., Phénix, T., \& Lemaire, B. (2016). Promoting the experimental dialogue between working memory and chunking: Behavioral data and simulation. Memory \& Cognition, 44, 420-434.

Portrat, S., \& Lemaire, B. (2015). Is refreshing in working memory sequential? A computational modeling approach. Cognitive Computation, 7, 333-345.

Raye, C. L., Johnson, M. K., Mitchell, K. J., Greene, E. J., \& Johnson, M. R. (2007). Refreshing: A minimal executive function. Cortex, 43, 135-45.

Raye, C. L., Johnson, M. K., Mitchell, K. J., Reeder, J. A., \& Greene, E. J. (2002). Neuroimaging a single thought: Dorsolateral PFC activity associated with refreshing just-activated information. NeuroImage, 15(2), 447-453.

Smith, E. E., \& Jonides, J. (1997). Working memory: a view from neuroimaging. Cognitive Psychology, 33, 5-42.

Tan, L., \& Ward, G. (2008). Rehearsal in immediate serial recall. Psychonomic Bulletin Review, 15, 535-42.

Vallar, G., \& Baddeley, A. D. (1982). Short-term forgetting and thearticulatory loop. Quarterly Journal of Experimental Psychology, 34A, 53-60. 
Vandierendonck, A. (2016). A working memory system with distributed executive control. Perspectives on Psychological Science, 11, 74 100

Vergauwe, E., Camos, V., \& Barrouillet, P. (2014). The impact of storage on processing: How is information maintained in working memory? Journal of Experimental Psychology: Learning, Memory \& Cognition, 40, 1072-1095.
Vergauwe, E., \& Cowan, N. (2015). Attending to items in working memory: Evidence that refreshing and memory search are closely related. Psychonomic Bulletin and Review, 22, 10011006.

Wagenmakers, E., \& Farrell, S. (2004). AIC model selection using Akaike weights. Psychonomic Bulletin \& Review, 11, 192196. 